\title{
Article \\ Effective Preventive Strategies to Prevent Secondary Transmission of COVID-19 in Hemodialysis Unit: The First Month of Community Outbreak in Taiwan
}

\author{
Chun-Yu Chen ${ }^{1,2}{ }^{1}$, Jung-Jr Ye ${ }^{3}$, Ting-Shuo Huang ${ }^{4,5} \oplus$, Chin-Chan Lee ${ }^{1,2}$, Yih-Ting Chen ${ }^{1,2}$, Cheng-Kai Hsu ${ }^{1,2}$, \\ Heng-Jung Hsu ${ }^{1,2}$, Chiao-Yin Sun 1,2, Heng-Chih Pan 1,2, Kuo-Su Chen ${ }^{1,2}$, Hao-Hsi Kao ${ }^{1,2}$, Chia-Chun Ko ${ }^{1,2}$, \\ Yun-Hsuan She ${ }^{1,2}$, Chun-Ying Wu ${ }^{6}$, Chi-Chun Lai ${ }^{2,7}$, Shang-Jyh Hwang ${ }^{8,9}$ and I-Wen $\mathrm{Wu}^{1,2, *}$
}

check for updates

Citation: Chen, C.-Y.; Ye, J.-J.; Huang, T.-S.; Lee, C.-C.; Chen, Y.-T.; Hsu, C.-K.; Hsu, H.-J.; Sun, C.-Y.; Pan, H.-C.; Chen, K.-S.; et al. Effective Preventive Strategies to Prevent Secondary Transmission of COVID-19 in Hemodialysis Unit: The First Month of Community Outbreak in Taiwan. Healthcare 2021, 9, 1173. https://doi.org/10.3390/ healthcare 9091173

\section{Academic Editors: Dimitrios}

Paraskevis, Maria Yavropoulou and Sotirios Tsiodras

Received: 17 August 2021

Accepted: 3 September 2021

Published: 7 September 2021

Publisher's Note: MDPI stays neutral with regard to jurisdictional claims in published maps and institutional affiliations.

Copyright: (c) 2021 by the authors. Licensee MDPI, Basel, Switzerland. This article is an open access article distributed under the terms and conditions of the Creative Commons Attribution (CC BY) license (https:/ / creativecommons.org/licenses/by/ $4.0 /)$.
1 Department of Nephrology, Chang Gung Memorial Hospital, Keelung 204, Taiwan; shone@cgmh.org.tw (C.-Y.C.); leefang@cgmh.org.tw (C.-C.L.); b9402031@cgmh.org.tw (Y.-T.C.); kylegb@cgmh.org.tw (C.-K.H.); r5267@cgmh.org.tw (H.-J.H.); sun3970@cgmh.org.tw (C.-Y.S.); balour@cgmh.org.tw (H.-C.P.); cksdavid@cgmh.org.tw (K.-S.C.); luhuichun@cgmh.org.tw (H.-H.K.); kinanna0815@cgmh.org.tw (C.-C.K.); she.yun.hsuan@gmail.com (Y.-H.S.)

2 College of Medicine, Chang Gung University, Taoyuan 333, Taiwan; ccl404@cgmh.org.tw

3 Department of Infectious Diseases, Chang Gung Memorial Hospital, Keelung 204, Taiwan; loyalwise@cgmh.org.tw

4 Community Medicine Research Center, Department of General Surgery, Chang Gung Memorial Hospital, Keelung 204, Taiwan; huangts@cgmh.org.tw

5 Department of Chinese Medicine, College of Medicine, Chang Gung University, Taoyuan 333, Taiwan

6 Department of Laboratory Medicine, Chang Gung Memorial Hospital, Keelung 204, Taiwan; hla0861@cgmh.org.tw

7 Department of Ophthalmology, Chang Gung Memorial Hospital, Keelung 204, Taiwan

8 Department of Internal Medicine, Division of Nephrology, Kaohsiung Medical University Hospital, Kaohsiung 807, Taiwan; sjhwang@kmu.edu.tw

9 Faculty of Renal Care, College of Medicine, Kaohsiung Medical University, Kaohsiung 807, Taiwan

* Correspondence: a22066@cgmh.org.tw; Tel.: +886-2-24313131-6211

Abstract: Background: Dialyzed patients are vulnerable to coronavirus infection disease 2019 (COVID-19). The incidence and outcome of COVID-19 in hemodialysis (HD) patients in Taiwan remain unclear. A series of preventive measures were executed to combat COVID-19 transmission among HD patients. Methods: We carried out a series of forward-looking and practical preventive strategies of COVID-19 control in our HD center. Incidences of COVID-19 of our HD unit were compared with those of national and local estimates from a community outbreak from 15 May to 30 June 2021. Prognostic factors associated with mortality were analyzed. Results: The national incidence of COVID-19 was $0.062 \%$; being highest in Taipei City $(0.173 \%)$, followed by New Taipei City $(0.161 \%)$ and Keelung $(0.083 \%)$. The overall incidence in Keelung HD patients was $0.666 \%$. One patient of our HD center contracted COVID-19 from the household; however, we have contained secondary transmission in our HD center by implementing strict preventive measures. The mortality rate of HD patients in Keelung was 66.6\%. The median Ct value of HD patients was 17.53 (11.75-27.90) upon diagnosis. The deceased patients had a higher cardiac/thoracic ratio than alive (0.61 vs. 0.55 , $p=0.036)$. Conclusions: Taking aggressive and proactive infection preventive measures impedes the secondary transmission of COVID-19 in HD facilities. COVID-19-associated mortality was high in HD patients, being the high cardiac-thoracic ratio, an important prognostic factor for clinical outcome of infected HD patients.

Keywords: COVID-19; incidence; mortality; outcome; prevention; strategies

\section{Introduction}

The 2019 novel severe acute respiratory syndrome coronavirus 2 (SARS-CoV-2) infection related disease (COVID-19) is extremely contagious, sometimes lethal in high-risk 
patients, and was declared a global emergency affecting $2.53 \%$ of the 7.8 billion population worldwide [1]. The disease is continuing to engender a tremendous global healthcare burden up to today. COVID-19 infection, which originated in Wuhan City of China in December 2019, has quickly spread to all continents, causing 197,825,365 confirmed cases and 4,216,780 deaths worldwide to 1 August 2021 [1]. Fortunately, the implementation of universal prevention and vaccination policies has led to a substantial reduction in the number of newly diagnosed cases in some countries, such as the United States, Israel, and the United Kingdom. However, the mortality rate remains high in other nations, including the United States, Brazil, and India [1]. Taiwan has fought against the disease and has achieved successful containment of the infection keeping up to 253 days of zero confirmed domestic cases since the beginning of the overwhelming global situation of the pandemic in 2020 [2]. The dissemination of infection has been prevented by using vigorous national control measures, such as country border restrictions, proactive case identification using electronic contact tracing, quarantine of suspicious and confirmed cases, enhancing hygiene and sanitation, mandatory masking, social distancing, healthcare resource allocation, and efforts in reassurance and education of citizens [3-5]. However, a second-wave outbreak of the COVID-19 pandemic has emerged in North Taiwan since 14 May 2021 and has spread into other regions of the country. Taiwan is attacked majorly by SARS-CoV-2 variant B.1.1.7 which originated from the United Kingdom; however, a small proportion of the delta variant, also known as B.1.617.2, was also noted [6]. The two virus variants are more transmissible, more virulent, and may trigger more grave clinical conditions than non-variants lineage $[7,8]$. As of 30 June, Taiwan has reached 14,804 laboratory-confirmed SARS-CoV-2 infection cases with an increasing death toll to 648 fatalities, especially in two hardest-hit areas, Taipei City and New Taipei City [9]. However, this community outbreak has been rapidly controlled nationwide. Sharing these experiences can certainly help in designing effective COVID-19 control measures in areas of similar socio-economic or epidemiological conditions.

End-stage renal disease (ESRD) patients represent a vulnerable population with increased risks for COVID-19 infection and for related death [10]. In-center hemodialysis (HD) is currently the most common renal replacement therapy modality for ESRD patients worldwide [11]. The regular needs of visiting a medical facility to receive their dialysis treatment, often confined in crowded spaces, make it difficult to comply with some of the effective protective measures, such as stay-home orders, social distancing, and reducing personal contacts. The risks of mortality in SARS-CoV-2 infected people include older age, presence of comorbidities, and immune dysfunction [12,13]. HD patients are often elderly and immune-dysregulated, owing to uremia, underlying comorbidities, and dialysis procedure-related biocompatibility and micro-inflammation [14]. Therefore, specific preventive methods to protect HD patients from COVID-19 infection remain urgently needed.

Experiences of effective preventive strategies to combat secondary transmission of COVID-19 in HD units remain limited. Keelung City, $30 \mathrm{~km}$ away from the hotspot area of the pandemic, has become a region of alert for the prevention of community spreading of the disease. Taking appropriate measures immediately in the HD unit is the top priority to hamper the dissemination of the disease. The common preventive strategies adopted in the HD unit comprise the use of independent ward or cohort isolation, hand sanitizing, quarantine, and effective personal protective equipment [10]. However, the infection rate remains still high in HD units in spite of the application of all these methods [10]. Taiwan has the highest prevalence of ESRD worldwide [11]; however, the estimation of incidence and outcome of COVID-19 infection in Taiwanese HD patients is unclear. In spite of national and official public health policies to fight against COVID-19, effective preventive strategies to impede the secondary transmission of COVID-19 in HD units remain to be established. The aim of this study was to design a series of preventive measures to fight against the spreading of SARS-CoV-2 infection among HD units and to evaluate the effectiveness of these measures on the incidence of COVID-19 and related prognosis of HD 
patients in this era of crisis. The information could be mandatory to limit the infection and dissemination of COVID-19 in HD facilities.

\section{Materials and Methods}

\subsection{Study Design and Subject Characteristics}

This study was conducted in a single hospital-affiliated HD center of Chang Gung Memorial Hospital at Keelung City, the largest HD unit of north-east Taiwan having 120 beds and caring for 497 maintenance HD patients. All of these patients were followed from the beginning of the community outbreak on 14 May 2021 to 30 June 2021. Demographics, laboratory data, and the incidence of COVID-19 were accurately recorded. All patients must comply with national public health preventive measures for the COVID-19 pandemic from the Taiwan Center for Disease Control (CDC) and several additional specific preventive strategies promulgated by our HD center. They have to agree to report their traveling fingerprint, including their traveling, occupational, contact, and clustering history (TOCC). The enrolled patients should be vaccinated in compliance with the government's COVID-19 vaccination program, except those with contraindications to vaccines. This study was performed in accordance with the Declaration of Helsinki and was approved by the Ethics Committee of the Institutional Review Board at Chang Gung Medical Foundation (IRB: 202100854B0A3).

\subsection{Formation of COVID-19 Joint Rapid Response Team}

A COVID-19 joint rapid response team from the HD Center in Chang Gung Memorial Hospital at Keelung was formed immediately after the outbreak of COVID-19 in Northern Taiwan on 15 May. The team included experts of nephrology and infectiology, leaders of HD nursing staff, nephrology nursing practitioners, members of the committee of infection control, and the chief person of the Administrative Department. All members of this multidisciplinary team joined the Team Pro ${ }^{\circledR}$ and LINE ${ }^{\circledR}$ group to facilitate immediate communication. The directors of the nephrology and infectiology department led the team and headed this collaborative effort. The COVID-19 joint rapid response team formulated our exclusive COVID-19 clinical infection preventive guidelines for HD patients and staff, arranged the acceptance of confirmed SARS-CoV-2 infection HD patients from outside clinical HD facilities, and connected extensively with the Department of Health of Keelung City Government to update daily infected cases. On 17 May 2021, the joint response team announced the first draft of the COVID-19 clinical practice guidelines for our HD Center, which were on the basis of recommendations from the Taiwan CDC and Taiwan Society of Nephrology (TSN) [15-18]. Our guidelines were targeted to prevent COVID-19 infection of HD patients, to minimize secondary spreading, and to establish response methods of our HD Center against the disease (Table 1).

Table 1. Clinical characteristics of maintenance hemodialysis patients in Chang Gung Memorial Hospital, Keelung Branch $(n=497)$.

\begin{tabular}{cc}
\hline & All HD Patients $(\boldsymbol{n}=\mathbf{4 9 7})$ \\
\hline Male, $n(\%)$ & $263(53)$ \\
Age, year & $66.03 \pm 13.28$ \\
White blood cell count $(\times 1000 / \mathrm{uL})$ & $6.62 \pm 2.87$ \\
Hemoglobin $(\mathrm{g} / \mathrm{dL})$ & $10.06 \pm 1.25$ \\
Platelet $(\times 1000 / \mathrm{uL})$ & $190.47 \pm 68.53$ \\
Albumin $(\mathrm{g} / \mathrm{dL})$ & $4.00 \pm 0.48$ \\
Aspartate transaminase $(\mathrm{IU} / \mathrm{L})$ & $18.49 \pm 10.31$ \\
Alanine aminotransferase $(\mathrm{IU} / \mathrm{L})$ & $17.87 \pm 14.74$ \\
Alkaline-P $(\mathrm{IU} / \mathrm{L})$ & $92(71-137){ }^{*}$ \\
Total bilirubin $(\mathrm{mg} / \mathrm{dL})$ & $0.38 \pm 0.16$ \\
Cholesterol $(\mathrm{mg} / \mathrm{dL})$ & $153.76 \pm 36.27$ \\
Triglyceride $(\mathrm{mg} / \mathrm{dL})$ & $151.77 \pm 116.22$ \\
Creatinine $(\mathrm{mg} / \mathrm{dL})$ & $9.40 \pm 2.68$ \\
\hline
\end{tabular}


Table 1. Cont.

\begin{tabular}{cc}
\hline & All HD Patients $(\boldsymbol{n}=\mathbf{4 9 7})$ \\
\hline Uric acid (mg/dL) & $6.43 \pm 1.82$ \\
$\mathrm{Na}(\mathrm{meq} / \mathrm{L})$ & $138.10 \pm 3.21$ \\
$\mathrm{~K}(\mathrm{meq} / \mathrm{L})$ & $4.70 \pm 0.83$ \\
$\mathrm{Ca}(\mathrm{mg} / \mathrm{dL})$ & $9.33 \pm 0.90$ \\
$\mathrm{P}(\mathrm{mg} / \mathrm{dL})$ & $5.26 \pm 1.55$ \\
Kt/V (Daugirdes) & $1.67 \pm 0.33$ \\
Ferritin (ng/mL) & $1.08 \pm 0.39$ \\
Normalized protein catabolic rate $(\mathrm{g} / \mathrm{kg} /$ day) & $496.46 \pm 415.14$ \\
Transferrin saturation $(\%)$ & $33.00 \pm 14.51$ \\
Intact-parathyroid hormone $(\mathrm{pg} / \mathrm{mL})$ & $285(119,672)^{*}$ \\
Cardiac/thoracic ratio & $0.52 \pm 0.07$ \\
Ca $\times$ P product & $49.17 \pm 15.67$ \\
\hline
\end{tabular}

${ }^{*}$ Data are expressed as the median (interquartile range).

\subsection{Specific Preventive Strategies in HD Center of Chang Gung Memorial Hospital at Keelung}

The preventive guidance issued by the TSN instructed universal measures for patients and medical staff to combat COVID-19, complimenting those interventions of the CDC [15-18]. The measures for patients were comprised of portal control, TOCC tracking and triaging, mandatory masking, health status, and temperature monitoring, restriction of eating and drinking in the treatment area during the whole HD session and limiting caregivers. In addition to all these measures, effective personnel protection equipment (PPE, using goggles, masks, gloves, isolation gowns) and frequent alcohol hand disinfection were recommended for all medical and nursing staff. For workplace safety, use of independent treatment rooms, appropriate environmental and equipment disinfection, especially in frequently touched areas by multiple people (toilet, doorknobs, elevator buttons, and others), maintenance of air-conditioning, and good ventilation conditions were suggested [16,19]. In spite of the use of these measures, outbreaks of COVID-19 infection were noted in different HD facilities nationwide. Furthermore, we implemented additional strategies to prevent the spreading of infection in our HD Center, focusing on the following aspects: space segmentation, access control, quarantine operations, and medical staff protection (Table 1). Most of the HD units used curtain separation rather than solid compartments between patients during their dialysis sessions. In addition to this universal measure, we utilized wood, strengthened glass, and acrylic materials to building small cabins (of 8-10 beds). This construction was safe, affordable, and rapid to reduce potential contacts and exposure to infected cases from the open space of the HD room (Figure 1). The entry to the HD unit, at each beginning of the treatment session, can cause clustering of patients in a crowded area during a concentrated period of time, which increases the risk of personal contacts and represents an important shortcoming for infection prevention. To overcome this disadvantage, we have established pacing between exchanges of HD sessions to avoid overlapping of patients at the entry and exit of HD rooms and have installed position marks on the ground of the entrance, with a $1.5 \mathrm{~m}$ distance between patients to avoid congregation (Figure 2A). We also installed acrylic partitions in the nursing station to limit droplet and aerosol dispersion (Figure 2B). Weekly rapid nasopharyngeal swab SARS-CoV-2 antigen testing was mandatory for all HD patients and fixed caregivers to promote early detection of asymptomatic cases. This maneuver has been conducted in an independent negative pressure room with the use of an auxiliary acrylic box and performed by physicians wearing complete PPE (Figure 2C) until a permanent positive-pressure cabinet became available (Figure 2D). The real-time PCR (RT-PCR) nucleic acid test was performed if a positive result from the rapid antigen test was noted. Suspected cases (or those waiting for RT-PCR results) received HD sessions in a negative pressure room via a separate gateway to enter our HD unit wore an N95 mask during the treatment session. All suspected cases were instructed to avoid public transportation until receiving negative RT-PCR results. For confirmed cases, patients were hospitalized in an independent negative pressure room and received bedside 
HD via portable reverse osmosis $(\mathrm{RO})$ system. The patients received at least 14-days of hospitalization until a negative RT-PCR result became available. Vaccination was highly promoted to all HD patients. We offered bedside inoculation at the end of their HD treatment. The beside vaccination program can incentivize the willingness to receive a vaccine and can also reduce further clustering at the vaccination station (Figure 2E,F). To avoid possible contamination of asymptomatic cases to our HD unit, we also developed triage algorithms, according to COVID-19 risk stratification, to manage those uremic patients presenting in the emergency room (ER) needing HD therapy (Figure 3). COVID-19 clinical practice guidelines raised by our joint rapid response team further instructed patients and staff to comply with all these measures. Periodic surveillances in the adherence of these interventions were conducted by a third party of the administrative department.

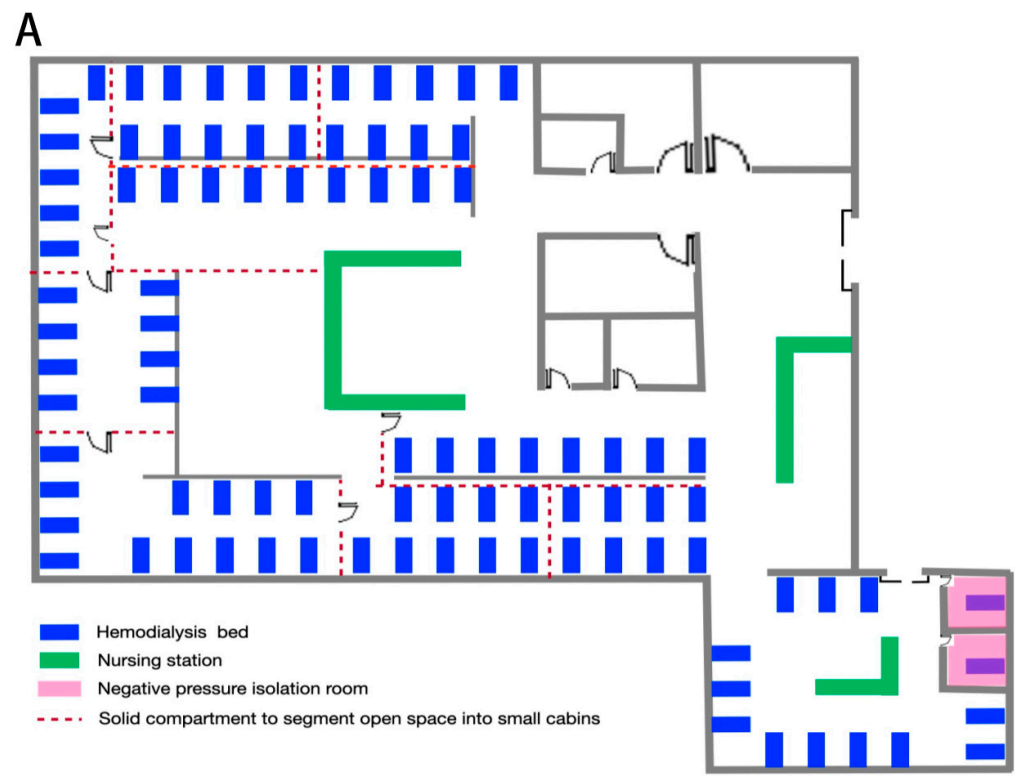

B

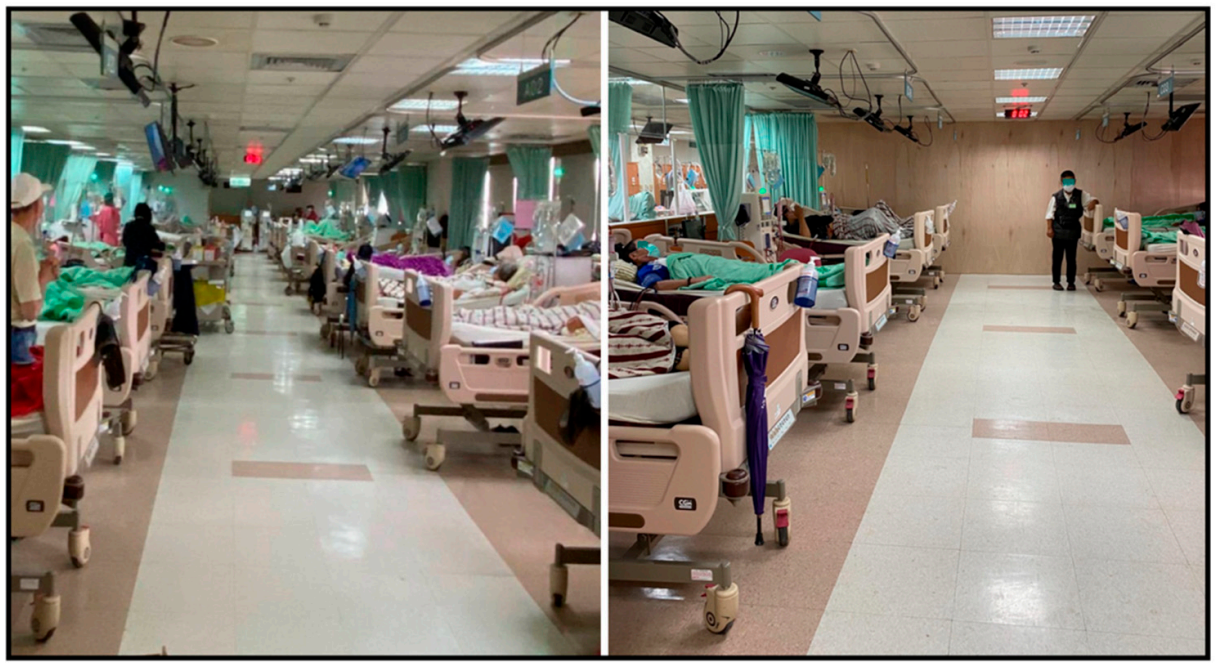

Figure 1. Space segmentation of HD unit of Chang Gung Memorial Hospital at Keelung. (A) Aerial view of space segmentation; (B) use of solid wood compartment to separate the open room into small cabins (left, before; right, after segmentation). 


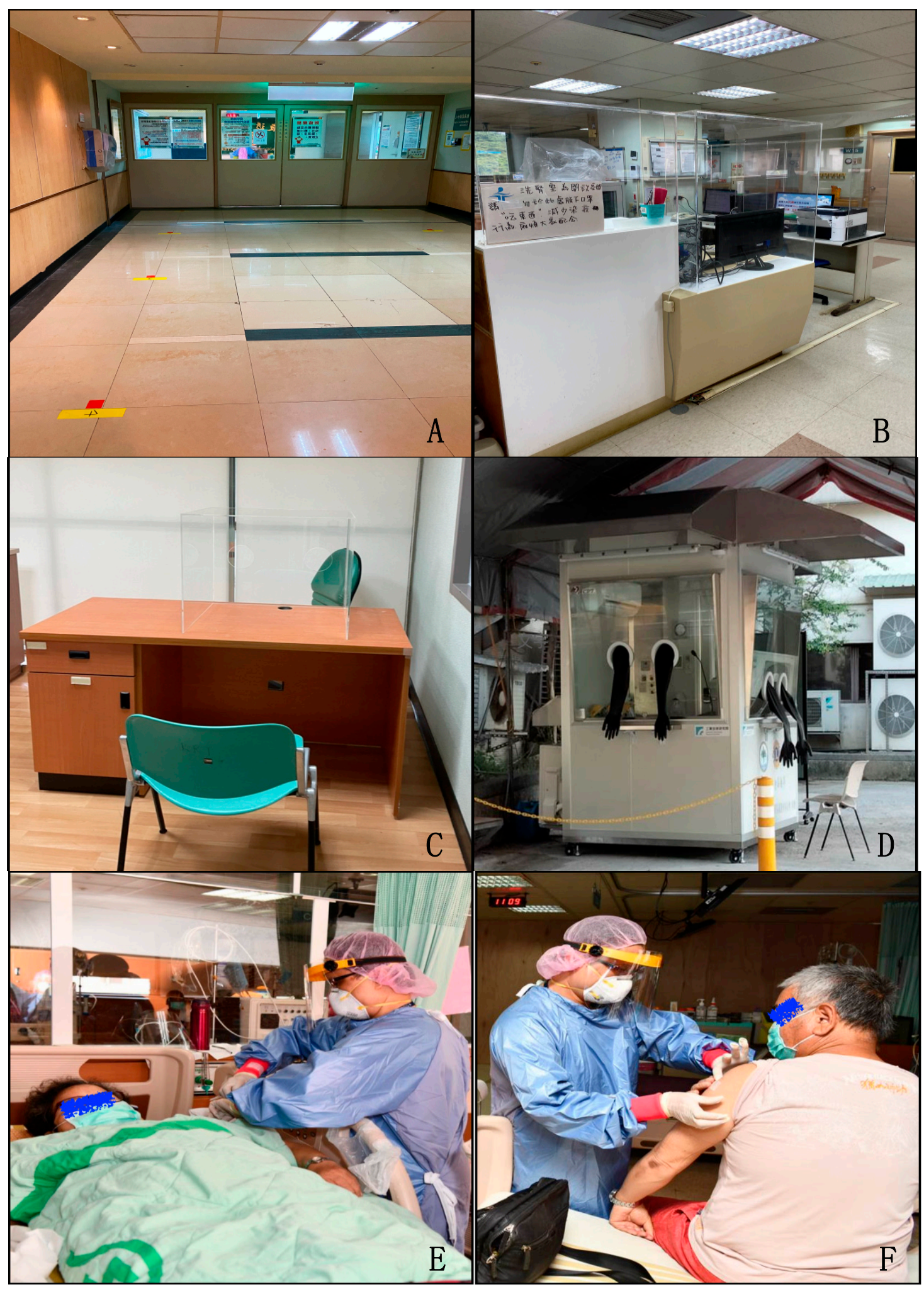

Figure 2. Specific preventive measures for COVID-19 infection control in the HD unit of Chang Gung Memorial Hospital at Keelung. (A) Set up position mark at entry of HD unit; (B) acrylics compartment for nursing station to prevent droplet dissemination; (C) weekly rapid test for early detection of asymptomatic patients at temporary setting or (D) permanent settings; bedside vaccination at HD unit during supine (E) or setting position (F). 
A

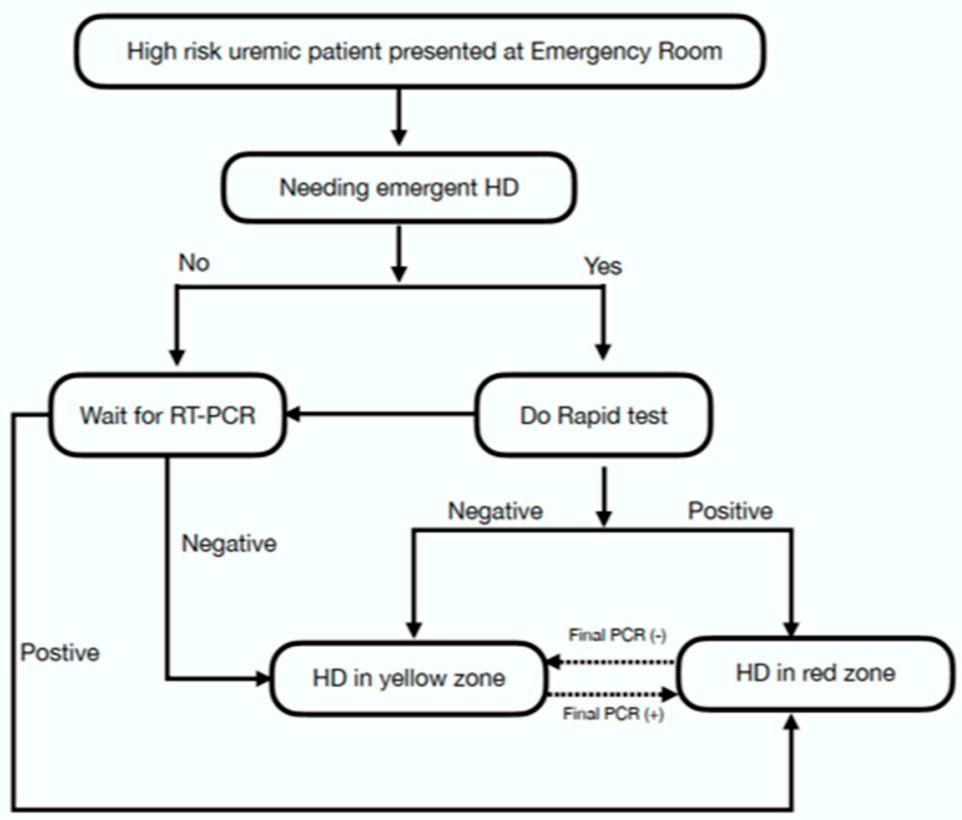

B

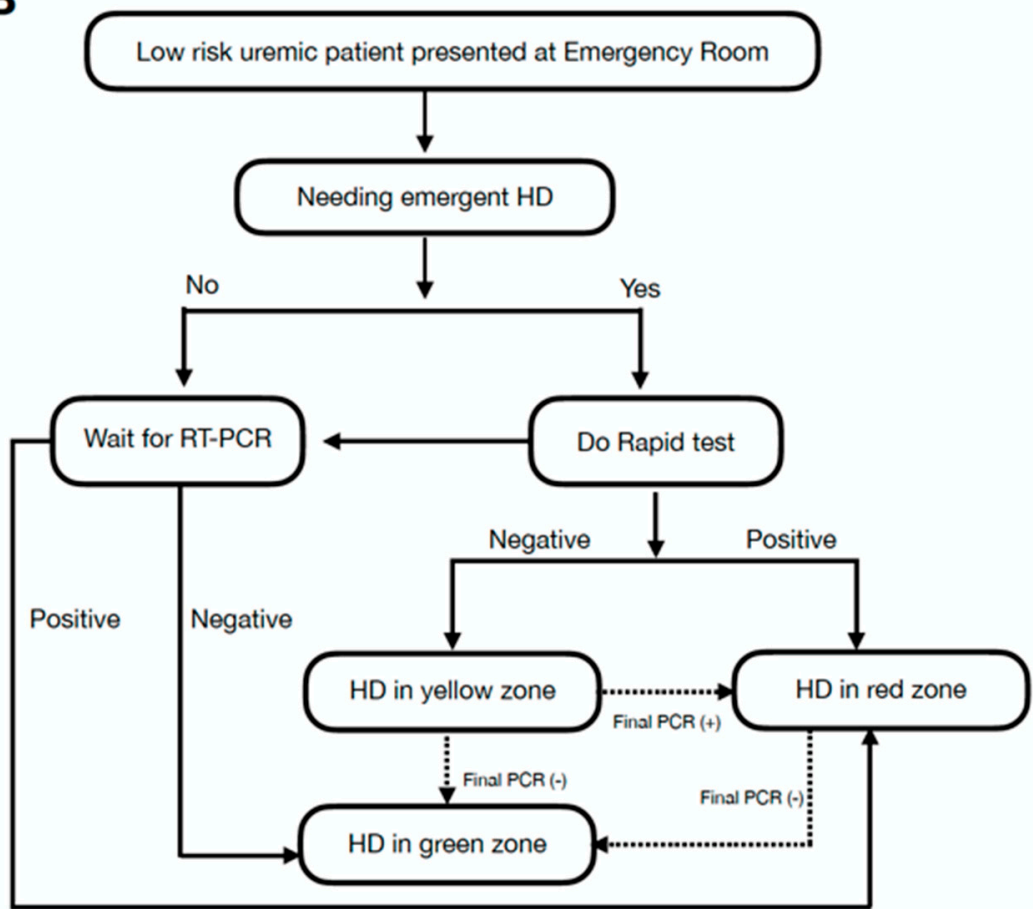

Figure 3. Triage algorithm of emergent patients needing HD during pandemics in Chang Gung Memorial Hospital at Keelung. (A) Algorithm for patients with high-risk of COVID-19, defined as having (1) recent home quarantine or self-health management (for less than 21 days), (2) community pneumonia, (3) resident of institutions, (4) any fever (ear temperature $\geq 38$ degrees), respiratory symptoms or diarrhea or abnormalities in taste or smell sensation, (5) recent exposure to confirmed patients; (B) algorithm for patients with low-risk of COVID-19 (who do not meet any of the abovementioned criteria). 


\subsection{Adjustment of Preventive Measures According to Reproduction Number $\left(R_{t}\right)$}

The transmission rate of COVID-19 infection in specific settings relies on the overall prevalence of disease of the region and can be estimated by the effective reproduction number (Rt) [20]. It is defined as the mean number of secondary cases generated by a typical primary case at a given time in a population and can be calculated for the whole period over a 5-day moving average [21]. The effective Rt can be used to evaluate the effectiveness of public health measures and should be quantified in different settings at regular and frequent intervals [22,23]. We adjusted our preventive plans according to the overall prevalence of COVID-19 in Keelung. We also stepped down our preventive measures guided by the Rt values (national estimates and those of the three high prevalent pandemic areas: Taipei City, New Taipei City, and Keelung) to achieve smooth recovery of normal medical functioning.

\subsection{Laboratory Confirmation for SARS-CoV-2 Infection: Rapid Antigen Test and RT-PCR Analysis}

We used the VTRUST COVID-19 Antigen Rapid Test (TaiDoc company, Taiwan) to detect SARS-CoV-2 nucleocapsid protein by using lateral flow chromatographic immunoassay on a weekly basis. The samples were obtained by nasopharyngeal swab specimen and results were obtained in $15 \mathrm{~min}$. The limit of detection (LoD) was $1.26 \times 102$ TCID50 per $\mathrm{mL}$ and the sensitivity and specificity were $93.1 \%$ and $99.6 \%$, respectively. Rapid test positive cases were further ascertained by nucleic acid testing using RT-PCR. Specimens for RT-PCR were obtained through nasopharyngeal swabs and immersed into $0.3 \mathrm{~mL}$ of preservation solution containing $30 \mu \mathrm{L}$ proteinase $\mathrm{K}$ and $0.33 \mathrm{~mL}$ VXL buffer. The multiplex RT-PCR (LabTurbo ${ }^{\mathrm{TM}}$ AIO COVID-19 RNA testing kit) was applied to detect SARS-CoV-2 RNA by using a full-process automated system. The multiplex reaction detected COVID-19 N1 gene, coronavirus E gene, and RNase P gene (internal control) in one single well. The COVID-19 RdRP gene was used for reconfirmation of the detection. The primer and probe sequences for RNA-dependent RNA polymerase gene detection were described as GTGARATGGTCATGTGTGGCGG (forward), CAAATGTTAAAAACACTATTAGCATA (reverse), and FAM-CAGGTGGAACCTCATCAGGAGATGC-BBQ (probe). Meanwhile, the primer and probe sequences used for $\mathrm{E}$ gene detection were ACAGGTACGTTAATAGTTAATAGCGT (forward), ATATTGCAGCAGTACGCACACA (reverse), FAM-ACACTAGCCATCCTTACTGCGCTTCG-BBQ (probe). The primer and probe sequences used for the N1 gene detection were as follows: GACCCCAAAATCAGCGAAAT (forward), TCTGGTTACTGCCAGTTGAATCTG (reverse), FAM-ACCCCGCATTACGTTTGGTGGACC-BBQ (probe). The cycle threshold (Ct) values of RT-PCR were converted into RNA copy number of SARS-CoV-2. The RNA copy number was calculated from a standard curve on the basis of the $\mathrm{Ct}$ values of plasmid DNA. The LoD of the quantitative $\mathrm{PCR}$ reaction for the $\mathrm{N} 1$ gene was 1 copy $/ \mu \mathrm{L}$, for the RdRP gene was 1 copy $/ \mu \mathrm{L}$, for the E gene was 2 copies $/ \mu \mathrm{L}$, and for the RNaseP gene was 0.1 copy $/ \mu \mathrm{L}$. Specimens were considered as negative if the $\mathrm{Ct}$ values exceeded 36 cycles. The technique for COVID-19 RNA detection is approved by the Taiwan Food and Drug Administration with emergent use authorization (EUA, approval number: 1096817658).

\subsection{Data Source of Local and National Burden}

The number of nationally confirmed cases and those of high prevalent areas, including Taipei City, New Taipei City, and Keelung, were recorded. The number of laboratoryconfirmed SARS-CoV-2 infections in Taiwan and of the individual counties or cities were obtained from the Taiwan CDC [9]. Information of COVID-19-infected HD patients in Keelung was obtained from the Taiwan CDC and the Daily Press conference of the Keelung City Government. The numbers of actual confirmed cases and the 7-day moving average confirmed cases were recorded for the analyses. 


\subsection{Statistical Analyses}

Descriptive statistics were expressed as the mean (standard deviation) or median (interquartile range). Discrete variables were presented in terms of frequencies and percentages. The normality of numerical variables was tested using the Kolmogorov-Smirnov method, and an appropriate transformation was considered before statistical testing. The Student's $t$-test or Mann-Whitney U-test was applied to compare the mean or median values among the groups. The association between categorical variables was analyzed using the chi-square test. We estimated the Rt values using the methods provided in the R package (EpiNow2, ver. 1.3.3) [21]. All statistical tests were two-tailed, and a $p$-value $<0.05$ was considered to be statistically significant. Data were analyzed using the statistical package from the social sciences software (SPSS, Inc., Chicago, IL, USA) version 26.0 for Mac.

\section{Results}

\subsection{Study Subject Characteristics}

Table 1 summarizes the clinical characteristics of our HD patients. The mean age was $66.03 \pm 13.28$ years, comparable to the average age of the national HD patients starting $\mathrm{HD}$. The mean hemoglobin was $10.06 \pm 1.25 \mathrm{~g} / \mathrm{dL}$ and the $\mathrm{Kt} / \mathrm{V}$ was $1.67 \pm 0.33$. The overall nutrition status was acceptable with a mean normalized protein catabolic rate of $1.08 \pm 0.39 \mathrm{~g} / \mathrm{kg} /$ day and albumin of $4.00 \pm 0.48 \mathrm{~g} / \mathrm{dL}$, respectively. The only SARS-CoV-2infected HD patient was younger (60.8 years), had less hemoglobin $(8.20 \mathrm{~g} / \mathrm{dL})$, less platelet count $(173 \times 1000 / \mathrm{uL})$, less albumin $(3.76 \mathrm{~g} / \mathrm{dL})$, less cholesterol $(139 \mathrm{mg} / \mathrm{dl})$, and higher intact-parathyroid hormone $(440 \mathrm{pg} / \mathrm{mL})$ values (Table S1).

\subsection{An Overview of the Pandemic in Northern Taiwan in the First Month of Outbreak}

Taiwan has maintained zero domestic infections for 253 days and had 12 deceased patients in 2020. However, a cluster of COVID-19 cases occurred in a hotel in Taoyuan County at the end of April 2021, imported by aircraft crews. After that, COVID-19 rapidly spread to northern Taiwan and finally broke out on 14 May 2021, with daily 185 newly diagnosed domestic cases. Taiwan has accumulated 14,804 confirmed cases and 648 deaths as of the end of June (Figure S1A). The northern cities were hit hardest by the pandemic. The national incidence of COVID-19 was $0.062 \%$; being highest in Taipei City $(0.173 \%)$, followed by New Taipei City $(0.161 \%)$ and Keelung $(0.083 \%)$, as of 30 June (Table 2$)$. The overall incidence of COVID-19 in Keelung HD patients was $0.666 \%$. One single HD patient contracted the COVID-19 infection from the household; however, we were able to contain secondary transmission of this case to our HD unit by using strict implementation and adherence to preventive measures. This community outbreak peaked in the period of 22-30 May in Taipei City, having daily 550 new confirmed cases (Figure S1B), and subsequently spread out to New Taipei City (Figure S1C) and Keelung. Bimodal waves of the pandemic were observed in Keelung with clustering in several marketplaces (Figure 4A). Fortunately, the pandemic has been contained with decremental cases nationwide over time (Figure S2A).

Table 2. Comparison between national and local incidence rates of SARS-CoV-2 infection and those of hemodialysis patients.

\begin{tabular}{|c|c|c|c|}
\hline Districts & Confirmed Cases & Total Population \# & Incidence Rate (\%) \\
\hline National & 14,804 & $23,816,775$ & 0.062 \\
\hline Taipei City & 4470 & $2,581,006$ & 0.173 \\
\hline New Taipei City & 6503 & $4,027,730$ & 0.161 \\
\hline Keelung City & 303 & 367,088 & 0.083 \\
\hline $\begin{array}{l}\text { Overall HD patients at } \\
\text { Keelung }\end{array}$ & 9 & 1351 & 0.666 \\
\hline CGMH-HDR * & 0 * & 497 & 0.000 \\
\hline
\end{tabular}

Abbreviations: CGMH-HDR, Chang Gung Memorial Hospital Keelung Branch hemodialysis room; HD, Hemodialysis. * Nosocomial infection. \# Data source: National Center for High-performance Computing, https:// covid-19.nchc.org.tw/city_confirmed.php (accessed on 1 August 2021). 


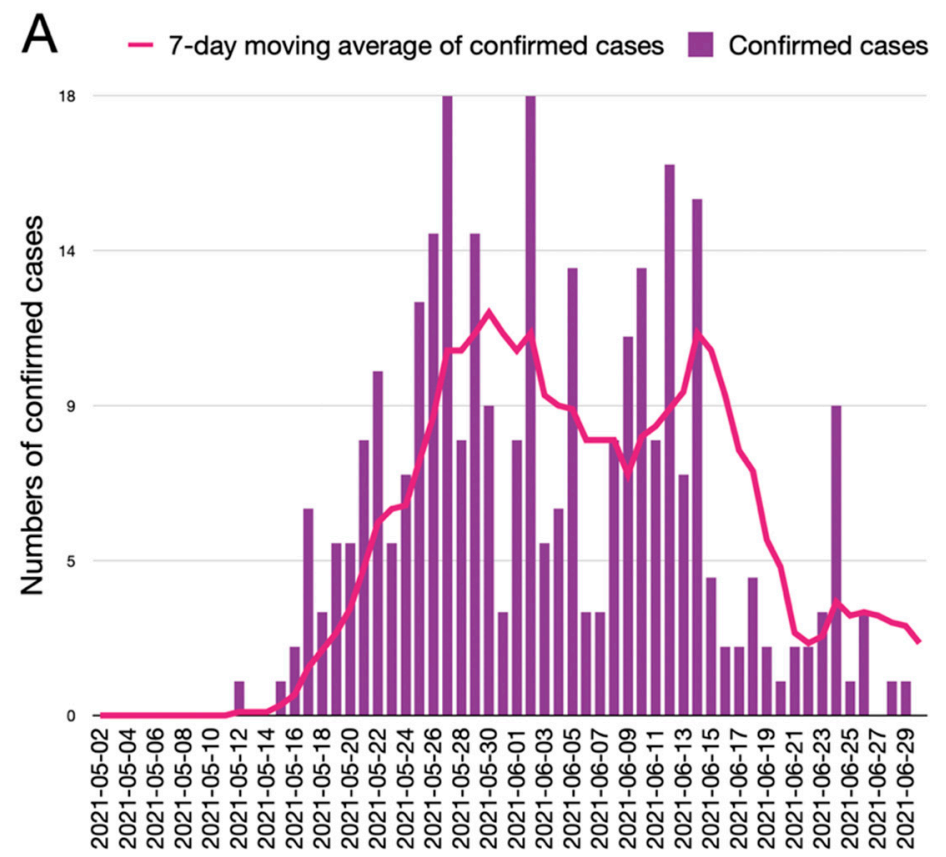

$\mathrm{B}$

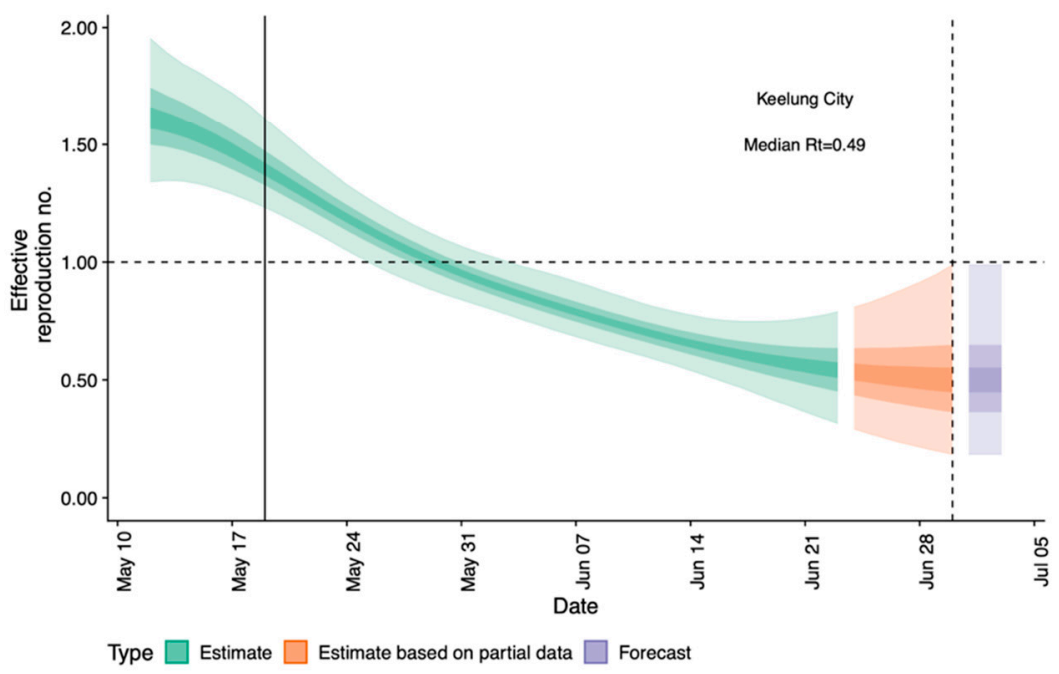

Figure 4. Estimates of COVID-19 confirmed case numbers and 7-day moving average of confirmed cases in Keelung (A). Estimated and predicted time-varying reproduction number (Rt) of SARS-CoV-2 with 20\%,50\%, 90\% credible intervals in Keelung (B).

\subsection{Outcomes of Strict Implementation of Specific Preventive Strategies in HD Unit}

Prevention of nosocomial spreading of airborne diseases, such as COVID-19, remains difficult in HD settings, especially in hotspot areas. To overcome this barrier, we have established specific preventive strategies for patient and staff safety (Table 3). One single patient of our HD unit contracted the COVID-19 infection from her family members. Confirmed HD cases or those with exposure to confirmed cases were moved to negative pressure isolation rooms for their dialysis treatment. Close contacts of these cases, including nursing staff and surrounding HD patients were all tested for RT-PCR. In spite of negative RT-PCR results, the nursing staff received home quarantine for two weeks and the surrounding HD patients were placed in a separated area for dialysis for 14 days. Fortunately, no new confirmed cases were noted after that episode, and we have successfully contained any secondary transmission of COVID-19 among our HD patients by using advanced specific 
preventive measures (Table 3). Although all of these measures were efficient and were rapidly implemented at the beginning of the outbreak; however, the interventions were both manpower- and resource-consuming and may hamper many other functioning of normal medical practice. The uses of our preventive measures were adjusted according to the regional prevalence and Rt values of the endemic region. The Keelung area exhibited the greatest reduction of Rt values over time (Figure 4B). Consequently, several measures (such as weekly rapid testing of our HD patients and caregivers) were suspended at six weeks after the initiation of the outbreak when the overall prevalence of Keelung dropped and the vaccination rate of our patients had reached more than $80 \%$ of the whole HD patient pool.

Table 3. Specific COVID-19 prevention measures of the Dialysis Center of CGMH, Keelung Branch in 2021.

\begin{tabular}{ll}
\hline Strategies & Contents of Measures \\
\hline & $\begin{array}{l}\text { Use of solid compartment to separate the open room into cabins of 8-10 beds, using wood, } \\
\text { strengthened glass, and acrylic as materials. }\end{array}$ \\
Space & $\begin{array}{l}\text { Use of acrylic partitions in the nursing station to limit droplet dissemination. } \\
\text { segmentation }\end{array}$ \\
$\begin{array}{l}\text { Fixed care bundle: fixed patients and caregivers in pre-established bed or seat location cared by a } \\
\text { fixed nursing team using fixed clinical carts. }\end{array}$ \\
$\begin{array}{l}\text { Installation of monitoring and loudspeaker system in negative pressure isolation room to limit } \\
\text { frequent entries and exits of personnel. }\end{array}$ \\
\hline
\end{tabular}

Spacing between exchanges of HD sessions to avoid overlapping of patients at entry and exit of HD room.

Double TOCC checking at both entry of hospital (by reading of NHI card) and at the door of HD unit (by personal verbal and written confirmation).

Access control Double-checking of clinical symptom and body temperature at both entry of hospital and at the door of HD unit.

Double hand alcohol disinfection at both entry of hospital and at door of HD unit.

Remove tables and chairs in the waiting area and set up a position mark on the ground of entry with $1.5 \mathrm{~m}$ of distancing to avoid clustering.

Control of the total number of people in the hospital and in a single elevator.

Postering and broadcasting (at entry of HD room, in the beginning of every HD session) of our HD preventive measures.

Quarantine Caregiver limitation (do not stay in treatment area, or fixed caregiver if assistance needed)

operations Weekly rapid nasopharyngeal swab SARS-CoV-2 antigen testing for patients and fixed caregivers.

Acceptance of new patients into our HD unit is prohibited during an outbreak. Triage algorithm

should be followed for emergent cases (see Figure 3).

Bedside vaccination program for all HD patients.

All medical and nursing staff wore high-quality PPE, including face shields, goggles, N95 masks, gloves, and waterproof isolation gowns.

Staff protection

Staff entering the dialysis room must perform a QR code scan to confirm location and contact

tracking.

Provide the first dose of COVID-19 vaccine to all of the staff before the end of May.

Staff who take care of confirmed patients should receive RT-PCR testing on a weekly basis.

All these measures were implemented in addition to those recommended by the Taiwan CDC and TSN. Abbreviations: HD, hemodialysis; TOCC, travel, occupation, contact, cluster history; NHI, national health insurance; PPE, personnel protection equipment; QR code, Quick Response code; COVID-19, coronavirus infection disease 2019; RT-PCR, real-time polymerase chain reaction.

\subsection{Outcomes of COVID-19 Confirmed HD Patients}

Two of the $497 \mathrm{HD}$ patients had persistent positive weekly rapid test results for more than 4 weeks; however, no presence of fever or clinical symptoms were noted in these two cases. The weekly RT-PCR testing for them was all negative. One single confirmed patient of our HD unit transmitted from the household developed severe pneumonia, while she responded well to therapy, survived, and was eventually discharged. In contrast, eight confirmed HD cases of infection were noted in a neighborhood HD unit at Keelung using 
only universal preventive measures of the CDC (Table 2). One of these patients died prior to admission to our hospital and five of them died after admission to our intensive care unit. Table 4 shows the characteristics of SARS-CoV-2 infection confirmed HD patients of Keelung City admitted to our hospital. Overall, nine confirmed HD cases were found at Keelung and the mortality rate was $66.6 \%$ (6 of 9). The median $C_{t}$ value of hospitalized patients was $17.53(11.75-27.90)$ upon diagnosis. The deceased patients tend to be older (74.9 vs. 63.1 years, $p=0.143)$, more likely having a higher cardiac/thoracic ratio (0.61 vs. $0.55, p=0.036)$ and higher serum C-reactive protein ( 52.8 vs. $17.2 \mathrm{mg} / \mathrm{L}, p=0.0711$ ). The serum levels of lactate ( 97.5 vs. $11.05, p=0.333)$, procalcitonin $(0.56$ vs. $0.53 \mathrm{ng} / \mathrm{mL}$, $p=0.393$ ), and $C_{t}$ value (17.47 vs. $\left.17.53, p=1.000\right)$ did not differ between deceased and alive patients, respectively (Table 4 ). All patients died from respiratory failure due to pneumonia having a median survival time of 13 (8-19) days from the date of diagnosis.

Table 4. Characteristics of COVID-19 confirmed HD patients in Keelung City $(n=8)$ *.

\begin{tabular}{|c|c|c|c|c|}
\hline & Total Patients $(n=8)$ * & Alive $(n=3)^{\$}$ & Deceased $(n=5)$ & $p$-Value \\
\hline Male, $n(\%)$ & $3(37.5)$ & $1(33.3)$ & $2(40)$ & 0.714 \\
\hline Age, year & $68.9(60.88-97.00)$ & $63.1(60.88-64.30)$ & $74.9(61-97)$ & 0.143 \\
\hline W.B.C. $(\times 1000 / \mathrm{uL})$ & $4.20(1.00-6.80)$ & $5.30(3.30-6.80)$ & $4.20(1.00-5.00)$ & 0.250 \\
\hline $\mathrm{Hb}(\mathrm{g} / \mathrm{dL})$ & $9.55(7.60-12.40)$ & $8.20(7.60-12.40)$ & $10.5(8.4-10.8)$ & 0.571 \\
\hline Platelet $(\times 1000 / \mathrm{uL})$ & $125(39-225)$ & $155(76-173)$ & $103(39-225)$ & 0.571 \\
\hline Albumin $(\mathrm{gm} / \mathrm{dL})$ & $3.66(3.06-4.15)$ & $3.76(3.27-3.91)$ & $3.56(3.06-4.15)$ & 1.000 \\
\hline AST (IU /L) & $25(16-67)$ & $26(16-61)$ & $24(20-67)$ & 1.000 \\
\hline ALT (IU/L) & $17(9-34)$ & $31(17-34)$ & $12.5(9-18)$ & 0.114 \\
\hline Alkaline-P (IU/L) & $110(48-154)$ & $128(48-154)$ & $110(69-110)$ & 0.700 \\
\hline Total Bilirubin (mg/dL) & $0.35(0.3-0.6)$ & $0.3(0.3-0.4)$ & $0.4(0.3-0.6)$ & 0.393 \\
\hline Cardiac/thoracic ratio & $0.59(0.48-0.65)$ & $0.55(0.48-0.57)$ & $0.61(0.58-0.65)$ & $0.036^{*}$ \\
\hline Creatinine (mg/dL) & $9.04(4.00-15.18)$ & $9.04(6.39-11.74)$ & $7.45(4-15.18)$ & 0.857 \\
\hline $\mathrm{Na}(\mathrm{meq} / \mathrm{L})$ & $137.5(121-141)$ & $138(137-139)$ & $135(121-141)$ & 0.393 \\
\hline $\mathrm{K}(\mathrm{meq} / \mathrm{L})$ & $3.70(3.2-6.9)$ & $4.60(3.20-5.70)$ & $3.70(3.4-6.90)$ & 1.000 \\
\hline $\mathrm{Ca}(\mathrm{mg} / \mathrm{dL})$ & $8.90(8.50-11.10)$ & $9.95(8.8-11.10)$ & $8.9(8.5-10.0)$ & 0.571 \\
\hline $\mathrm{P}(\mathrm{mg} / \mathrm{dL})$ & $5.00(2.00-9.90)$ & $4.40(2.8-6.0)$ & $5.0(2.0-9.9)$ & 1.000 \\
\hline Ferritin (ng/mL) & $869.5(41.9-6954.0)$ & $877.0(41.9-1100.0)$ & $862.0(82.3-6954.0)$ & 0.786 \\
\hline $\mathrm{Ca} \times \mathrm{P}$ product & $38.54(20-88.11)$ & NA (31.08-52.80) & $46.00(20.00-88.11)$ & 0.571 \\
\hline Ct value & $17.50(11.75-27.10)$ & $17.53(11.75-27.10)$ & 17.47 (16.75-18.57) & 1.000 \\
\hline CRP (mg/L) & 36.62 (15.17-70.79) & $17.2(15.17-35.70)$ & $52.81(20.60-70.79)$ & 0.071 \\
\hline LDH $(\mathrm{U} / \mathrm{L})$ & $249(185-355)$ & $249(185-355)$ & $243.5(219.0-290.0)$ & 1.000 \\
\hline Lactate $(\mathrm{mg} / \mathrm{dL})$ & $46.35(7.80-116.60)$ & $11.05(7.80-14.30)$ & $97.5(78.40-116.60)$ & 0.333 \\
\hline Procalcitonin (ng/mL) & $0.535(0.16-1.75)$ & $0.51(0.16-0.56)$ & $0.56(0.48-1.75)$ & 0.393 \\
\hline
\end{tabular}

Abbreviation: AST, aspartate transaminase; ALT, alanine aminotransferase; HD, hemodialysis; LDH, lactate dehydrogenase; WBC, white blood cell count; $\mathrm{Ct}$ value, threshold cycle for the measure of the concentration of target in the polymerase chain reaction for SARS-CoV-2 nucleic acid testing. ${ }^{*}$ Overall confirmed 9 HD patients were noted in Keelung City. One patient died before admission to our hospital and only 8 patients were described in the present study. ${ }^{\$}$ One single patient in our HD unit transmitted from the household is one of these patients.

\section{Discussion}

In the present study, we have demonstrated that taking aggressive and proactive infection preventive measures could successfully impede secondary transmission of COVID-19 in HD facilities. The nosocomial spreading was greater in HD units than in other settings because of the high susceptibility of HD patients to SARS-CoV-2 infection. This vulnerability was inherent to the particular clinical- and treatment-related characteristics of dialysis patients. The national government has issued a level-3 alert since 17 May and has suspended school classes, limited the number of gatherings, prohibited businesses in entertainment venues, and forbidden meals in restaurants. Our COVID-19 joint rapid response team managed COVID-19 control through collaborative activities and has formulated clinical infection preventive guidelines for our HD center from the very early 
stage of the outbreak. In addition to national preventive measures, strict implementation and adherence of four major specific strategies: space segmentation, access control, quarantine operations, and high-quality medical staff protection have shown advantages to fighting against SARS-CoV-2 infection and have ensured the safety of patients and staff during their hemodialysis treatment, even in the presence of confirmed cases. However, COVID-19-associated mortality is high in HD patients, being the high cardiac-thoracic ratio, an important prognostic factor for clinical outcomes of infected HD patients.

Coronaviruses, commonly transmitted human-to-human, have caused several disastrous pandemics in human history. The outbreak of severe acute respiratory syndrome (SARS) in 2003 resulted in 346 confirmed SARS cases and 37 deaths in Taiwan. Nonspecific clinical presentation, absence of accurate and timely diagnostic methods, as well as a lack of experience of national prevention control measures in facing an emergency of a highly contagious airborne disease has led to nosocomial transmission and a catastrophic outbreak of the SARS epidemic in Taiwan [24]. Both SARS and Middle East respiratory syndrome (MERS) coronavirus infections have never contributed to secondary transmission in HD facilities [25-27]. Similarly, the prevalence of COVID-19 among HD settings has been higher than in the general population. Our previous systematic review and meta-analysis study has revealed that the overall incidence of COVID-19 in HD patients was 7.7\%, the incidence rate being lower in Asian HD patients (5.0\%, 95\% CI: $2.5-8.4 \%)$ than those of non-Asian countries (10.5\%, 95\% CI: 6.6-15.3\%) [10]. Although the national estimates of the incidence of COVID-19 in HD patients remain unclear, we have shown that the overall incidence rate of COVID-19 confirmed HD patients at Keelung was $0.666 \%$, 10-fold higher than the national COVID-19 infection estimate of the general population. Episodes of secondary transmissions were noted in various HD units of Wan-Hua District (Taipei City), New Taipei City, Keelung, and many other areas in May 2021. In one of the HD units, one confirmed HD patient resulted in the propagation of disease into seven other HD patients and four medical staff members. The tremendous outcome of five deaths was noted in that episode. Effective prevention of the nosocomial spreading of SARS-CoV-2 among HD settings remains mandatory.

In spite of the exhibition of a high incidence rate of COVID-19 in HD patients (at Keelung) than the incidence of national citizens, this rate has remained low compared to international estimates of the HD population [10,28-33]. HD patients are more susceptible to SARS-CoV-2 infection because of older age, the coexistence of multiple comorbidities, and immune-suppressed status. Necessary and periodic visits to areas of high population density (public transportation or HD facilities) and close personal contacts (with caregivers, medical or nursing staff) make effective strategies to prevent COVID-19, such as social distancing or stay-home orders, difficult to execute for this vulnerable patient population [34-36]. However, our preparedness for fighting against the disease today is better than the experiences of the previous years in many other countries, where uncertainties arose at the beginning of the global pandemic. The learning of valuable information regarding disease prevention from various nephrology societies and experts over the past years has been extremely helpful. In addition, the relatively low confirmed domestic cases in our territory [9], early national level-3 alert measures, universal masking, early formulation of clinical practice guidelines for fighting of COVID-19 from the CDC, and TSN have all contributed to the lower incidence of our HD patients compared to the international burden of infection in the dialysis population [37]. From official guidelines, good indoor air ventilation, stringent environment cleaning, and disinfection are mandatory for HD settings. All HD patients must wear a mask during dialysis therapy. Other measures consist of stage-based, proactive screening using TOCC tracking, traffic control bundle and checkpoint hand washing, fixed caring model (including divided work team to care fixed group of patients) to minimize the staff-patient exposures [18]. The guidelines of TSN have further enforced tasks for leadership, education, preparedness, proactive management, and a recovery stage for fighting against COVID-19 [18]. We have carried forward and enhanced all of these recommendations in practice. For leadership, our rapid response 
team enabled immediate, confident, and transparent communication between staff; for education, periodic updates of informative posters and regular broadcasting to both the medical team and patients were performed in our HD unit every day; for preparedness and proactive management, a series of strategies focusing on space segmentation, access control, quarantine operations, and high-quality medical staff protection were built to optimize prevention control; ultimately, for the recovery stage, we stepped down our preventive plans, guided by using serial assessments of Rt values and regional prevalence rates, to restore the healthcare capacity and resource utilization into normal medical functioning. The COVID-19 vaccination program was very effective and has helped to achieve an inoculation rate of $82 \%$ to mid-July in our HD units. The application of all these policies was possible to contain COVID-19 transmission in our HD unit maintaining zero cases of infection to this day since the initiation of community outbreak in Taiwan.

The high mortality rate $(66.6 \%)$ in COVID-19-infected HD patients in Keelung was peculiar. Analyses of the outcomes of COVID-19 infection have revealed overall mortality of $22.4 \%$ (ranged from 8.9 to $43.9 \%$ ) in various HD patient cohorts reported in 2020 [10]. Common prognostic factors for mortality in COVID-19-infected HD patients included greater age, male gender, underlying comorbidities (cardiac or pulmonary disease, diabetes, and hypertension), and the use of mechanical ventilation, corticosteroids, or wheelchairs $[36,38,39]$. In addition to all these factors, the increased chest-thoracic ratio was also a poor prognostic predictor among our HD patients, highlighting the importance of fluid control and underlying cardiac performance in improving outcomes. Furthermore, the mortality of the second wave of the outbreak affected by COVID variants was higher than the initial global pandemic [40]. HD patients infected with SARS-CoV-2 variants B.1.362 (from Israel) were more likely to have critical COVID-19 (defined as a condition associated with organ failure requiring ventilation or hemodynamic support), shorter survival time, and poorer outcome, having a mortality rate as high as $57 \%$ than the nonvariants COVID-19-infected HD patients [40]. Information regarding the outcome of HD patients infected with the SARS-CoV-2 variant B.1.1.7 (from the UK), the main virus strain in Taiwan, remains scarce. Large populational cohort studies revealed that patients infected with variants B.1.1.7 had a greater hospitalization rate, higher risk for intensive care, and higher 28-day mortality compared with patients with non-B.1.1.7 SARS-CoV-2 [41,42]. The emergency of SARS-CoV-2 variants has again emphasized the importance of infection control in HD settings because of the increased infectivity, virulence, and adverse outcome associated with these virus stains.

The HD unit of Chang Gung Memorial Hospital at Keelung is the largest renal care center in the region. We have successfully contained the COVID-19 infection and no secondary transmission was noted, despite the presence of one confirmed HD case infected from the household. National universal measures were rapidly implemented in addition to our specific preventive strategies from the beginning of the outbreak. Although we have provided a successful care model in preventing COVID-19 transmission in an HD facility, several works should be further addressed. First, many of our specific preventive plans were both resource- and time-consuming and may not be applicable to other private HD settings or areas of low prevalence rates. Our HD unit is a hospital-affiliated setting caring for approximately $500 \mathrm{HD}$ patients, in which multidisciplinary infrastructure and medical resources are adequate to support all of the measures. The devotion of personnel to prevention controls may hamper a certain proportion of normal healthcare functions; however, through the use of high-quality PPE to protect our HD staff and the periodic evaluation of regional Rt values, it was capable of preserving medical manpower during the pandemic and allowed the gradual recovery of normal functioning leveraging extreme preventive policies. Second, the use of weekly rapid testing screening may have underestimated the exact incidence of disease due to the insufficient power of testing kits. The employment of double-checking of TOCC, clinical symptoms, and body temperature (at both entry of the hospital and at the door of the HD room) may help to identify suspected cases in a timely fashion. In addition, compliance and adherence to preventive 
plans represent key components of any disease control. Appropriate education, through postering and broadcasting, regular surveillance, and monitoring from the third-party administrative department may further consolidate the establishment of our preventive strategies. Finally, the emergence of new SARS-CoV2 variants and changes to immunity in the post-vaccination era may also arise new challenges in the care of HD patients in facing a global pandemic. COVID-19 remains less likely to be eliminated in the near future, persistent alerts, monitoring, and efforts in disease prevention may become new norms for the daily clinical care of renal patients.

\section{Conclusions}

In conclusion, we have demonstrated a real-world experience of combating the spread of COVID-19 in an HD unit situated in a hotspot area of the outbreak. This infection was associated with a high mortality rate. In addition to national universal prevention policies, specific preventive strategies such as space segmentation, access control, quarantine operations, and high-quality medical staff protection may enhance many tasks recommended by TSN to create a safe and clean medical area for HD patients and staff.

Supplementary Materials: The following are available online at https://www.mdpi.com/article /10.3390/healthcare9091173/s1, Figure S1: Estimates of COVID-19 confirmed case number and 7-days moving average of confirmed cases. (A) National estimates, (B) in Taipei City and (C) in New Taipei City. Figure S2: Estimated and predicted time-varying reproduction number (Rt) of SARS-CoV-2 using national and subnational case counts reported in Taiwan Centers for Disease Control between 2 May 2021, and 30 June 2021. Estimation of time-varying Rt with 20\%, 50\%, 90\% credible intervals using national data in Taiwan (A), Taipei City (B) and New Taipei City (C). Table S1: Clinical characteristics of non-COVID and COVID patients on maintenance hemodialysis in Keelung Chang Gung Memorial Hospital $(n=497)$.

Author Contributions: Writing_original draft, Formal analysis, Project administration, Resources, C.-Y.C.; conceptualization, Methodology, J.-J.Y.; software, data curation, formal analysis, T.-S.H.; investigations, C.-C.L. (Chin-Chan Lee), Y.-T.C., C.-K.H., H.-J.H., C.-Y.S., H.-C.P., K.-S.C., H.-H.K., C.-C.K. and Y.-H.S.; data curation, validation, C.-Y.W.; resources, supervision, C.-C.L. (Chi-Chun Lai); visualization, supervision, S.-J.H.; supervision, conceptualization, writing-review and editing, project administration, funding acquisition, I.-W.W. All authors have read and agreed to the published version of the manuscript.

Funding: This research was funded by Chang Gung Memorial Hospital, grant number: CGRPG2F0081 and CRRPG2H0123.

Institutional Review Board Statement: The study was conducted according to the guidelines of the Declaration of Helsinki, and approved by the Institutional Review Board of Chang Gung Medical Foundation. The approval number is 202100854B0A3.

Informed Consent Statement: Informed consent was obtained from all subjects involved in the study.

Data Availability Statement: Not applicable.

Acknowledgments: We thank Taiwan Society of Nephrology in releasing practice guideline recommendations for fighting against COVID-19. We also express our gratitude to all of the nephrology medical and nursing staff members for their dedicated efforts during the COVID-19 epidemic; Department of Laboratory Medicine, for sophisticated rapid antigen and nucleic acid testing; Committee of infection control, for suggestions of infection prevention and Department of Administration for excellent supports in medical equipment, manpower and resource allocation.

Conflicts of Interest: The authors declare no conflict of interest.

\section{References}

1. WHO. Coronavirus Disease (COVID-19) Dashboard. Available online: https:// covid19.who.int/ (accessed on 1 August 2021).

2. National Center for High-Performance Computing. Daily New Diagnoses in Each County, City and Region. Available online: https://covid-19.nchc.org.tw/_ity_confirmed.php?mycity (accessed on 1 August 2021). 
3. Cheng, S.-Y.; Wang, C.J.; Shen, A.C.-T.; Chang, S.-C. How to safely reopen colleges and universities during COVID-19: Experiences from Taiwan. Ann. Intern. Med. 2020, 173, 638-641. [CrossRef] [PubMed]

4. $\quad$ Cheng, H.-Y.; Jian, S.-W.; Liu, D.-P.; Ng, T.-C.; Huang, W.-T.; Lin, H.-H.; The Taiwan COVID-19 Outbreak Investigation Team. Contact tracing assessment of COVID-19 transmission dynamics in Taiwan and risk at different exposure periods before and after symptom onset. JAMA Intern. Med. 2020, 180, 1156-1163. [CrossRef]

5. Wang, C.J.; Ng, C.; Brook, R.H. Response to COVID-19 in Taiwan: Big data analytics, new technology, and proactive testing. JAMA 2020, 323, 1341. [CrossRef] [PubMed]

6. WHO. Weekly Epidemiological Update on COVID-19. Available online: https://www.who.int/docs/default-source/coronaviru se/situation-reports/20210720_weekly_epi_update_49.pdf?sfvrsn=e036283e_4\&download=true (accessed on 20 July 2021).

7. Davies, N.G.; Jarvis, C.I.; Edmunds, W.J.; Jewell, N.P.; Diaz-Ordaz, K.; Keogh, R.H. Increased mortality in community-tested cases of SARS-CoV-2 lineage B.1.1.7. Nat. Cell Biol. 2021, 593, 270-274. [CrossRef]

8. Davies, N.G.; Abbott, S.; Barnard, R.C.; Jarvis, C.I.; Kucharski, A.J.; Munday, J.D.; Pearson, C.A.B.; Russell, T.W.; Tully, D.C.; Washburne, A.D.; et al. Estimated transmissibility and impact of SARS-CoV-2 lineage B.1.1.7 in England. Science 2021, 372, eabg3055. [CrossRef]

9. Taiwan Centers for Disease Control. COVID-19 Statistics of Taiwan. Available online: https://sites.google.com/cdc.gov.tw/2019 ncov / taiwan?authuser=0 (accessed on 15 January 2020).

10. Chen, C.-Y.; Shao, S.-C.; Chen, Y.-T.; Hsu, C.-K.; Hsu, H.-J.; Lee, C.-C.; Sun, C.-Y.; Chen, Y.-C.; Hung, M.-J.; Wu, I.-W. Incidence and clinical impacts of COVID-19 infection in patients with hemodialysis: Systematic review and meta-analysis of 396,062 hemodialysis patients. Healthcare 2021, 9, 47. [CrossRef] [PubMed]

11. United States Renal Data System. 2020 USRDS Annual Data Report: Epidemiology of Kidney Disease in the United States; National Institutes of Health: Bethesda, MD, USA; National Institute of Diabetes and Digestive and Kidney Diseases: Bethesda, MD, USA, 2020.

12. Zhou, F.; Yu, T.; Du, R.; Fan, G.; Liu, Y.; Liu, Z.; Xiang, J.; Wang, Y.; Song, B.; Gu, X.; et al. Clinical course and risk factors for mortality of adult inpatients with COVID-19 in Wuhan, China: A retrospective cohort study. Lancet 2020, 395, $1054-1062$. [CrossRef]

13. Rao, K.-S.; Suryaprakash, V.; Senthilkumar, R.; Preethy, S.; Katoh, S.; Ikewaki, N.; Abraham, S.J.K. Role of immune dysregulation in increased mortality among a specific subset of COVID-19 patients and immune-enhancement strategies for combatting through nutritional supplements. Front. Immunol. 2020, 11, 1548. [CrossRef]

14. Betjes, M.G.H. Immune cell dysfunction and inflammation in end-stage renal disease. Nat. Rev. Nephrol. 2013, 9, 255-265. [CrossRef] [PubMed]

15. Taiwan Centers of Disease Control. Guidelines on Infection Control Measures for Medical Institutions with Hemodialysis Facilities in Response to COVID-19. Available online: https: / www.cdc.gov.tw / File/Get/Cvg5lnAeQ2ySM301hyg6pw (accessed on 1 August 2021).

16. Li, Y.-J. Prolong of Level 3 Alert, Recommendations for Medical Responses of Dialysis Facilities in Taiwan. Available online: https: / / www.tsn.org.tw / tsnFile/authority/F8D926898A85F502/\%E5\%BB\%B6\%E9\%95\%B7\%E4\%B8\%89\%E7\%B4\%9A\%E8\% AD $\%$ A6\%E6\%88\%92\%20\%E5\%85\%A8\%E5\%8F\%B0\%E9\%80\%8F\%E6\%9E\%90\%E9\%99\%A2\%E6\%89\%80\%E9\%86\%AB\%E7\%99 \%82\%E8\%B3\%87\%E6\%BA\%90\%E6\%95\%B4\%E5\%82\%99\%E6\%87\%89\%E8\%AE\%8A\%E5\%BB\%BA\%E8\%AD\%B0_20210530_\% E7\%AC\%AC\%E4\%B8\%89\%E7\%89\%88.pdf (accessed on 10 August 2021). (In Chinese).

17. Lee, J.-J.; Hwang, S.-J.; Huang, J.-F. Review of the present features and the infection control challenges of COVID-19 pandemic in dialysis facilities. Kaohsiung J. Med. Sci. 2020, 36, 393-398. [CrossRef]

18. Lee, J.-J.; Lin, C.-Y.; Chiu, Y.-W.; Hwang, S.-J. Take proactive measures for the pandemic COVID-19 infection in the dialysis facilities. J. Formos. Med. Assoc. 2020, 119, 895-897. [CrossRef] [PubMed]

19. COVID-19 Emergency Response Team. Specifications of Dialysis Facilities Prevent COVID-19 Management Specifications. Available online: https:/ / www.tsn.org.tw/tsnFile/authority/F8D7DD61F9E8EC28/20200406-\%E9\%80\%8F\%E6\%9E\%90\%E9 \%86\%AB $\%$ E7\%99\%82\%E9\%99\%A2\%E6\%89\%80\%E5\%9B\%A0\%E6\%87\%89\%E6\%AD\%A6\%E6\%BC\%A2\%E8\%82\%BA\%E7\%82 \%8ECOVID-19\%E6\%84\%9F\%E6\%9F\%93\%E8\%99\%95\%E7\%BD\%AE\%E8\%A6\%8F\%E7\%AF\%84(\%E5\%85\%AC\%E5\%91\%8A\%E 7\%89\%88\%E7\%AC\%AC\%E4\%B8\%89\%E7\%89\%88)-TSN.pdf (accessed on 12 August 2021). (In Chinese).

20. Inglesby, T.V. Public health measures and the reproduction number of SARS-CoV-2. JAMA 2020, 323, 2186. [CrossRef] [PubMed]

21. Abbott, S.; Hellewell, J.; Thompson, R.N.; Sherratt, K.; Gibbs, H.P.; Bosse, N.I.; Munday, J.D.; Meakin, S.; Doughty, E.L.; Chun, J.Y.; et al. Estimating the time-varying reproduction number of SARS-CoV-2 using national and subnational case counts [version 1; peer review: Awaiting peer review]. Wellcome Open Res. 2020, 5, 112. [CrossRef]

22. Rubin, D.; Huang, J.; Fisher, B.T.; Gasparrini, A.; Tam, V.; Song, L.; Wang, X.; Kaufman, J.; Fitzpatrick, K.; Jain, A.; et al. Association of social distancing, population density, and temperature with the instantaneous reproduction number of SARS-CoV-2 in counties across the United States. JAMA Netw. Open 2020, 3, e2016099. [CrossRef] [PubMed]

23. Pan, A.; Liu, L.; Wang, C.; Guo, H.; Hao, X.; Wang, Q.; Huang, J.; He, N.; Yu, H.; Lin, X.; et al. Association of public health interventions with the epidemiology of the COVID-19 outbreak in Wuhan, China. JAMA 2020, 323, 1915-1923. [CrossRef]

24. Hsueh, P.R.; Yang, P.C. Severe acute respiratory syndrome epidemic in Taiwan, 2003. J. Microbiol. Immunol. Infect. 2005, $38,82-88$.

25. Kwan, B.C.-H.; Leung, C.-B.; Szeto, C.-C.; Wong, V.W.-S.; Cheng, Y.-L.; Yu, A.W.-Y.; Li, P.K.-T. Severe acute respiratory syndrome in dialysis patients. J. Am. Soc. Nephrol. 2004, 15, 1883-1888. [CrossRef] [PubMed] 
26. Assiri, A.; McGeer, A.; Perl, T.M.; Price, C.S.; Al Rabeeah, A.A.; Cummings, D.A.; Alabdullatif, Z.N.; Assad, M.; Almulhim, A.; Makhdoom, H.; et al. Hospital outbreak of middle east respiratory syndrome coronavirus. N. Engl. J. Med. 2013, 369, 407-416. [CrossRef]

27. Ahmed, A.E.; Alshukairi, A.N.; Al-Jahdali, H.; Alaqeel, M.; Siddiq, S.S.; Alsaab, H.A.; Sakr, E.A.; Alyahya, H.A.; Alandonisi, M.M.; Subedar, A.T.; et al. Development of a risk-prediction model for Middle East respiratory syndrome coronavirus infection in dialysis patients. Hemodial. Int. 2018, 22, 474-479. [CrossRef]

28. Albalate, M.; Arribas, P.; Torres, E.; Cintra, M.; Alcázar, R.; Puerta, M.; Ortega, M.; Procaccini, F.; Martin, J.; Jiménez, E.; et al. High prevalence of asymptomatic COVID-19 in haemodialysis: Learning day by day in the first month of the COVID-19 pandemic. Nefrología 2020, 40, 279-286. [CrossRef]

29. Wang, R.; Liao, C.; He, H.; Hu, C.; Wei, Z.; Hong, Z.; Zhang, C.; Liao, M.; Shui, H. COVID-19 in hemodialysis patients: A report of 5 cases. Am. J. Kidney Dis. 2020, 76, 141-143. [CrossRef] [PubMed]

30. Corbett, R.W.; Blakey, S.; Nitsch, D.; Loucaidou, M.; McLean, A.; Duncan, N.; Ashby, D.R.; The West London Renal and Transplant Center. Epidemiology of COVID-19 in an Urban Dialysis Center. J. Am. Soc. Nephrol. 2020, 31, 1815-1823. [CrossRef] [PubMed]

31. Kikuchi, K.; Nangaku, M.; Ryuzaki, M.; Yamakawa, T.; Hanafusa, N.; Sakai, K.; Kanno, Y.; Ando, R.; Shinoda, T.; Nakamoto, H.; et al. COVID-19 of dialysis patients in Japan: Current status and guidance on preventive measures. Ther. Apher. Dial. 2020, 24, 361-365. [CrossRef] [PubMed]

32. La Milia, V.; Bacchini, G.; Bigi, M.C.; Casartelli, D.; Cavalli, A.; Corti, M.; Crepaldi, M.; Limardo, M.; Longhi, S.; Manzoni, C.; et al COVID-19 outbreak in a large hemodialysis center in Lombardy, Italy. Kidney Int. Rep. 2020, 5, 1095-1099. [CrossRef] [PubMed]

33. Cho, J.-H.; Kang, S.H.; Park, H.C.; Kim, D.K.; Lee, S.-H.; Do, J.Y.; Park, J.W.; Kim, S.N.; Kim, M.S.; Jin, K.; et al. Hemodialysis with cohort isolation to prevent secondary transmission during a COVID-19 outbreak in Korea. J. Am. Soc. Nephrol. 2020, 31, 1398-1408. [CrossRef]

34. Wang, H. Maintenance hemodialysis and COVID-19: Saving lives with caution, care, and courage. Kidney Med. 2020, 2, 365-366. [CrossRef]

35. Su, K.; Ma, Y.; Wang, Y.; Song, Y.; Lv, X.; Wei, Z.; Shi, M.; Ding, G.; Shen, B.; Wang, H. How we mitigated and contained the COVID-19 outbreak in a hemodialysis center: Lessons and experience. Infect. Control Hosp. Epidemiol. 2020, 41, 1240-1242. [CrossRef]

36. Alberici, F.; Delbarba, E.; Manenti, C.; Econimo, L.; Valerio, F.; Pola, A.; Maffei, C.; Possenti, S.; Lucca, B.; Cortinovis, R.; et al. A report from the brescia renal COVID task force on the clinical characteristics and short-term outcome of hemodialysis patients with SARS-CoV-2 infection. Kidney Int. 2020, 98, 20-26. [CrossRef]

37. Akbarialiabad, H.; Kavousi, S.; Ghahramani, A.; Bastani, B.; Ghahramani, N. COVID-19 and maintenance hemodialysis: A systematic scoping review of practice guidelines. BMC Nephrol. 2020, 21, 1-9. [CrossRef]

38. Valeri, A.M.; Robbins-Juarez, S.Y.; Stevens, J.S.; Ahn, W.; Rao, M.K.; Radhakrishnan, J.; Gharavi, A.G.; Mohan, S.; Husain, S.A. Presentation and outcomes of patients with ESKD and COVID-19. J. Am. Soc. Nephrol. 2020, 31, 1409-1415. [CrossRef]

39. Hsu, C.M.; Weiner, D.E.; Aweh, G.; Miskulin, D.C.; Manley, H.J.; Stewart, C.; Ladik, V.; Hosford, J.; Lacson, E.C.; Johnson, D.S.; et al. COVID-19 among US dialysis patients: Risk factors and outcomes from a national dialysis provider. Am. J. Kidney Dis. 2021, 77, 748-756. [CrossRef] [PubMed]

40. Wand, O.; Mor, O.; Zuckerman, N.; Fadeela, A.; Benchetrit, S.; Nacasch, N.; Cohen-Hagai, K. Outcomes from infections with variant strains of SARS-CoV-2 among patients receiving maintenance hemodialysis. Am. J. Kidney Dis. 2021. [CrossRef]

41. Nyberg, T.; Twohig, K.A.; Harris, R.J.; Seaman, S.R.; Flannagan, J.; Allen, H.; Charlett, A.; De Angelis, D.; Dabrera, G.; Presanis, A.M. Risk of hospital admission for patients with SARS-CoV-2 variant B.1.1.7: Cohort analysis. BMJ 2021, 373, n1412. [CrossRef] [PubMed]

42. Patone, M.; Thomas, K.; Hatch, R.; Tan, P.S.; Coupland, C.; Liao, W.; Mouncey, P.; Harrison, D.; Rowan, K.; Horby, P.; et al. Mortality and critical care unit admission associated with the SARS-CoV-2 lineage B.1.1.7 in England: An observational cohort study. Lancet Infect. Dis. 2021. [CrossRef] 